\title{
Phase Separation Process in Polymer Systems. I. Light Scattering Studies on a Polystyrene and Poly(methylphenylsiloxane) Mixture
}

\author{
Shuichi NoJIMA, Katsuaki TsuTsumI, and Takuhei NOSE \\ Department of Polymer Chemistry, Tokyo Institute of Technology, \\ Ookayama, Meguro-ku, Tokyo 152, Japan.
}

(Received September 16, 1981)

\begin{abstract}
Early stages of phase separation in liquid mixtures of polystyrene (PS) and poly(methylphenylsiloxane) were studied by light scattering. Measurements were made at four concentrations less than $60 \mathrm{wt} \%$ PS and at several temperatures in the two-phase region. The time $(t)$ dependence of maximum intensity of scattered light $I_{\mathrm{m}}$ and the corresponding wave number $k_{\mathrm{m}}$ did not obey the Cahn theory but could be represented by power-laws: $I_{\mathrm{m}} \propto t^{\theta}$ and $k_{\mathrm{m}} \propto t^{\phi}$ with $\theta=$ $0.69( \pm 0.17)$ and $\phi=-0.29( \pm 0.05)$. These values of $\theta$ and $\phi$ agree well with those predicted by recent theories of Langer et al. and Binder et al. for spinodal decomposition, and also with the experimental values for binary mixtures of small molecules. It was concluded that there is no essential difference in the mechanism of phase separation between mixtures of small molecules and polymer blends.
\end{abstract}

KEY WORDS Phase Separation / Polymer Blend / Light Scattering / Spinodal Decomposition / Cahn's Theory /

A phase separation of polymer systems upon quenching starts from a homogeneous state and proceeds through a series of heterogeneous states, as is the case with the liquid-gas phase transition in one-component systems or with liquid-liquid or solid-solid phase separation in two-component systems of small molecules. The process can be divided into two main stages, early and late, depending on the degree of time evolution. ${ }^{1}$

At the early stages, there occur dominant concentration fluctuations in a homogeneous phase (mother phase or matrix), and these lead to the birth of new phases (daughter phases). At the late stages, called the coarsening process, ${ }^{2}$ the size and number of daughter phases change accompanying neither nucleation of new phases nor variation in composition, and the gradual aggregation of daughter phases occurs by what is called the coalescence ${ }^{1}$ or Ostwald Ripening mechanism..$^{3,4}$ Finally, the entire system settles down to a macroscopic two-phase state.

The early stage proceeds either by spinodal decomposition (SD) or by nucleation and growth. ${ }^{5}$
The former takes place spontaneously without energy barriers when the system is brought into an unstable region, since any concentration fluctuation over a certain range of wave lengths reduces the free energy of the unstable system. The latter starts when the system enters into a metastable region, in which the system is unstable only for seldom-occurring sufficiently large concentration fluctuations. Thus, in this, there is an energy barrier for the nucleation of a new phase, but once it is formed, the new phase grows rapidly by diffusion.

Spinodal decomposition was first formulated by Hillert $^{6}$ and Cahn (Cahn theory) $)^{7}$ based on a linear mean field theory for concentration fluctuations. ${ }^{8}$ In the framework of a mean field approximation, the Cahn theory was modified by Cook, ${ }^{9}$ Langer, ${ }^{10-12}$ and Abraham $^{13,14}$ to take non-linear effects into account, and extended to polymer blends by de Gennes with the tube model..$^{15}$ On the other hand, Binder ${ }^{16}$ and Kawasaki and Ohta ${ }^{17}$ have developed new approaches to spinodal decomposition by using modern theories of phase transition.

Recently, many experimental studies have been 
made for the spinodal decomposition of liquid mixtures of small molecules. ${ }^{18-26}$ For polymer solutions and polymer blends, however, only a few experimental studies on SD have been made, ${ }^{27-31}$ while some studies have focused on coarsening processes. ${ }^{32,33}$ For a liquid mixture of polystyrene and poly(vinyl methyl ether), for example, Nishi et $a l .{ }^{30}$ observed by pulsed NMR technique the change in composition with time during spinodal decomposition and evaluated the kinetic parameters describing the spinodal decomposition by use of the Cahn theory.

In this series of studies, our aim has been to obtain a understanding of the mechanisms of phase separation, at both the early and late stages, of polymer solutions and polymer blends. The present paper reports a light scattering study on the early stage of phase separation in liquid mixtures of polystyrene (PS) and poly(methylphenylsiloxane) (PMPS). This system was chosen for the following three reasons. First, the cloud points and glass transition points of this system appear in comparable ranges of temperature (see our forthcoming paper ${ }^{34}$ ). Hence, the separation rate is expected to be so slow that the early stage of phase separation can be conveniently followed by light scattering. Second, the two polymers are so close in density that gravity effects can be minimized. Third, the condition that their refractive indices are also very close to each other is favorable for light scattering experiment.

\section{EXPERIMENTAL}

\section{Materials and Sample Preparation}

The polystyrene (PS) sample was a product of Pressure Chemical Co. Its weight-average molecular weight $M_{w}$ and the ratio of $M_{w}$ to the numberaverage molecular weight $M_{n}$ were 9,000 and less than 1.06 , respectively. The values of density $d$ and refractive index $n$ of this material at room temperature were evaluated to be $1.07 \mathrm{~g} \mathrm{~cm}^{-3}$ and 1.59 , respectively. ${ }^{35,36}$ The poly(methylphenylsiloxane) (PMPS) was obtained from Toray Silicone Co. Its $M_{w}$ and $M_{w} / M_{n}$ determined by gel permeation chromatography were 2,800 and 1.57 , respectively. The values of $d$ and $n$ of this material at room temperature were $1.10 \mathrm{~g} \mathrm{~cm}^{-3}$ and 1.55 , respectively. Before use, PMPS was dried under vacuum for $50 \mathrm{~h}$ at $110^{\circ} \mathrm{C}$ to eliminate impurities.
Blends of PS and PMPS were prepared by two methods depending on the PS concentration. At higher PS concentrations, the two polymers were dissolved in a common solvent benzene and the solvent was evaporated at $110^{\circ} \mathrm{C}$ for $50 \mathrm{~h}^{37} \mathrm{At}$ lower PS concentrations, the two polymers were directly mixed with no solvent. The blends prepared by these methods gave a consistent cloud point curve.

\section{Measurements}

Intensities of light scattered from a given blend at angles from $0^{\circ}$ to $40^{\circ}$ were detected as voltages from a photodiode. A $0.5 \mathrm{~mW} \mathrm{He}-\mathrm{Ne}$ laser, of vacuum wavelength $\lambda_{0}=6328 \AA$, was used as the light source. A cell with an optical path as short as 0.1 $\mathrm{mm}$ was used to reduce multiple scattering. A linear relationship was found to hold between the voltage and scattered intensity. It took about $2 \mathrm{~min}$ to scan the angular range $0^{\circ}-40^{\circ}-0^{\circ}$. Scattered intensities at different angles were corrected for the measuring time and the time lag of the photodiode. Turbidity corrections made using the decrease in transmitted light intensity were about $70 \%$ for 23.9 wt $\%$ PS and $10 \%$ for $50.4 \mathrm{wt} \%$ PS at most. Real scattering angle $\theta$ was calculated from measured angle $\theta^{\prime}$ by $\sin \theta=\left(\sin \theta^{\prime}\right) / n$, where $n$ is the refractive index of the blend.

Phase separation was allowed to occur by quenching the blend from a temperature just above the cloud point to the desired temperature $T_{0}$. In all cases examined, it took about $1 \mathrm{~min}$ for the blend to reach thermal equilibrium at $T_{0}$. Changes in scattered intensity were followed as a function of time for $240 \mathrm{~min}$ except for this initial period after quenching.

As illustrated in Figure 1, the cloud points and glass transition points of the present system appeared in comparable ranges of temperature. This fact allowed us to expect that the mobility of polymers is so suppressed in the temperature region under consideration that phase separation should proceed very slowly. In fact, for the blend of $60 \mathrm{wt} \%$ PS, no appreciable proceeding of phase separation was observed in our experimental time scale and the cloud point depended strongly on the cooling rate. Thus, our light scattering experiment was limited to four blends with PS concentrations between 24 and $55 \mathrm{wt} \%$, and for each of these the data were taken at several $T_{0}$ in the range of $10^{\circ} \mathrm{C}$ below the cloud 


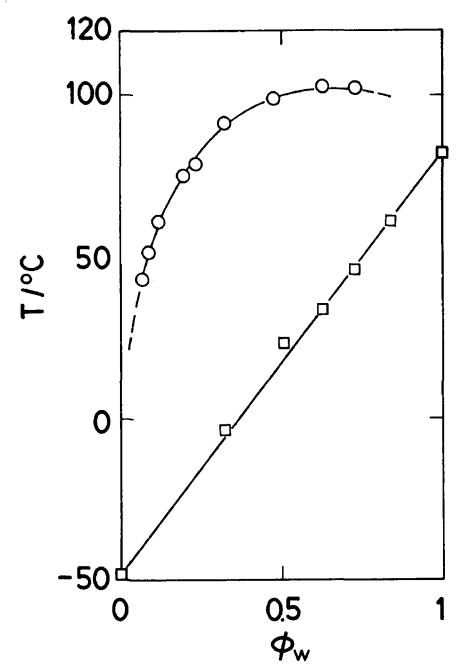

Figure 1. Cloud points $(O)$ and glass transition points ( $\square$ ) of PS-PMPS mixture.

point curve.

\section{RESULTS}

\section{General Features of Scattered Intensity}

Figure 2 shows the scattering pattern for 39.5 $\mathrm{wt} \% \mathrm{PS}$ and $\Delta T$ of $3.7^{\circ} \mathrm{C}$ taken at $50 \mathrm{~min}$ after quenching. Here $\Delta T$ is the quench depth from the cloud point temperature $T_{\mathrm{c}}(0)$. It delineates a characteristic ring of scattered light called the "spinodal ring". This ring appeared almost immediately after quenching, decreased in size, increased in intensity, and finally collapsed to a spot of an unscattered beam as phase separation proceeded. This phenomenon is essentially similar to those observed in spinodal decompositions of binary mixtures of small molecules. ${ }^{18-26}$ Another feature of Figure 2 is the granular pattern of the ring, which is also similar to the photograph taken by Goldburg et al. for the critical mixture of $2-6$ lutidine and water, ${ }^{20}$ and is considered to be characteristic of spinodal decomposition. This type of pattern was detected as small fluctuations in voltage $V$ plotted against scattering angle $\theta$, as shown in Figure 3.

Figure 4 illustrates for $50.4 \mathrm{wt} \%$ PS and $\Delta T=$ $6.7^{\circ} \mathrm{C}$ the time evolution of scattered intensity $I$ as a function of the wave number $k$. Here $k$ is defined by

$$
k=\left(4 \pi n / \lambda_{0}\right) \sin (\theta / 2)
$$

We see that the maximum intensity $I_{\mathrm{m}}$ increases very

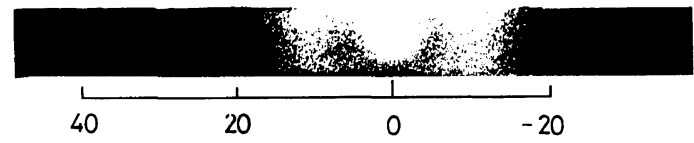

Figure 2. Scattering pattern from the blend of 39.5 $\mathrm{wt} \% \mathrm{PS}$ at a quench depth $\Delta T=3.7^{\circ} \mathrm{C}$ and a time $t=50$ min after quenching.

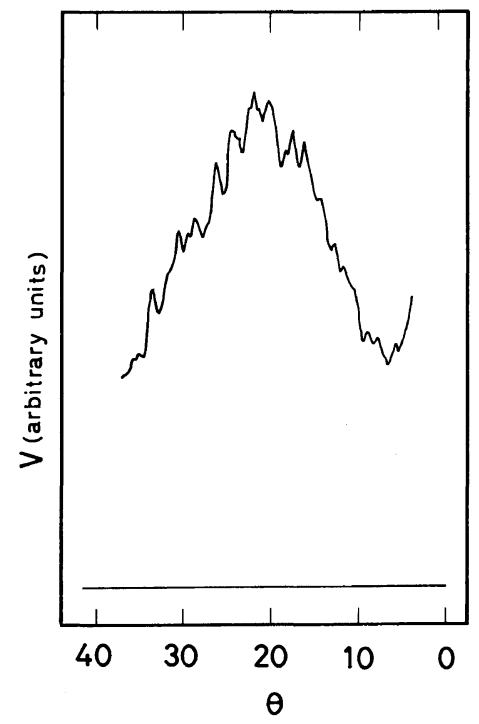

Figure 3. Voltage $V$ of a photodiode as a function of scattering angle $\theta$.

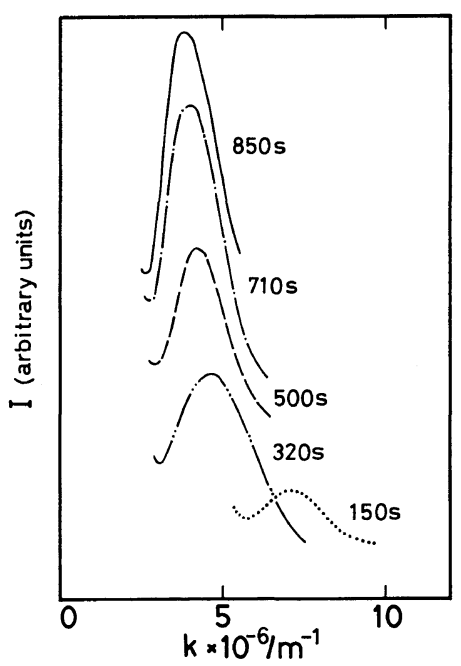

Figure 4. Scattered intensity $I$ as a function of $k$ and $t$ for a blend of $50.4 \mathrm{wt} \% \mathrm{PS}$ at $\Delta T=6.7^{\circ} \mathrm{C}$. 


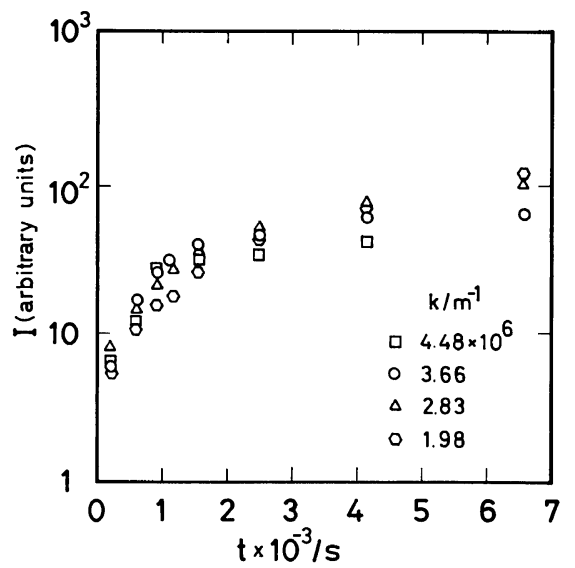

Figure 5. Scattered intensity $I$ as a function of $t$ for a blend of $50.4 \mathrm{wt} \%$ PS at $\Delta T=6.7^{\circ} \mathrm{C}$.

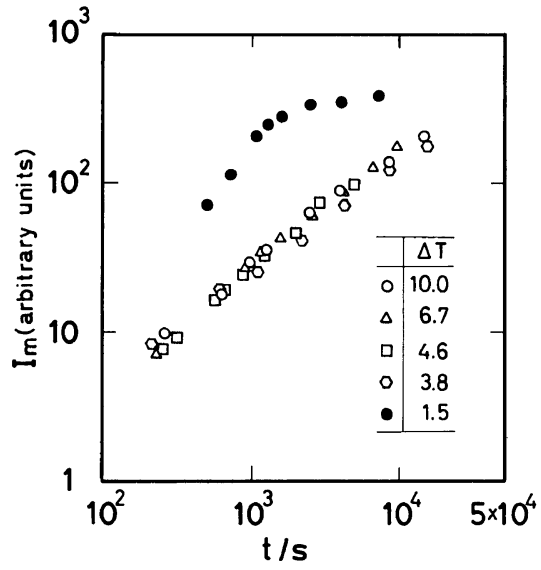

Figure 6. Maximum intensity $I_{\mathrm{m}}$ as a function of $t$ for a blend of $50.4 \mathrm{wt} \%$ PS at various $\Delta T$.

rapidly with time, while the corresponding wave number $k_{\mathrm{m}}$ decreases slowly. In Figure 5, the values of $I$ at four $k$ are plotted against time.

Time Dependence of $I_{\mathrm{m}}$ and $k_{\mathrm{m}}$

Figure 6 shows the time dependence of $I_{\mathrm{m}}$ for the blend of $50.4 \mathrm{wt} \%$ PS at different $\Delta T$, and Figure 7 shows the corresponding plot for the blend of 23.9 $\mathrm{wt} \%$ PS. In the former we see that, except for $\Delta T=$ $1.5^{\circ} \mathrm{C}$, the data points for different $\Delta T$ approximately fall on a single straight line. This behavior is not seen in the latter, although the points for individual $\Delta T$ again follow a straight line. When compared at the same time, the values of $I_{\mathrm{m}}$ for

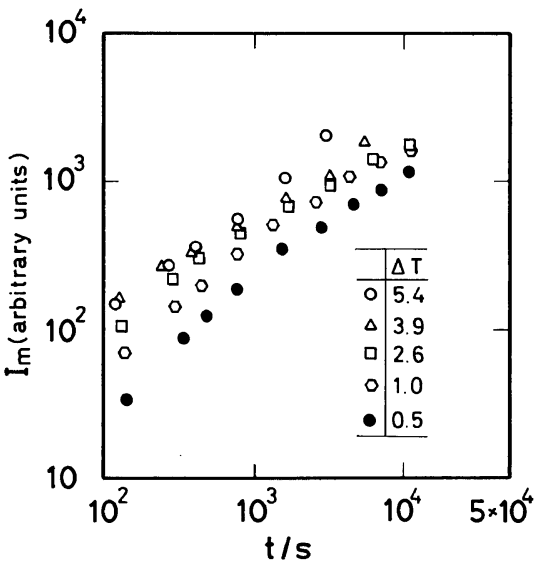

Figure 7. Maximum intensity $I_{\mathrm{m}}$ as a function of $t$ for a blend of $23.9 \mathrm{wt} \%$ PS.

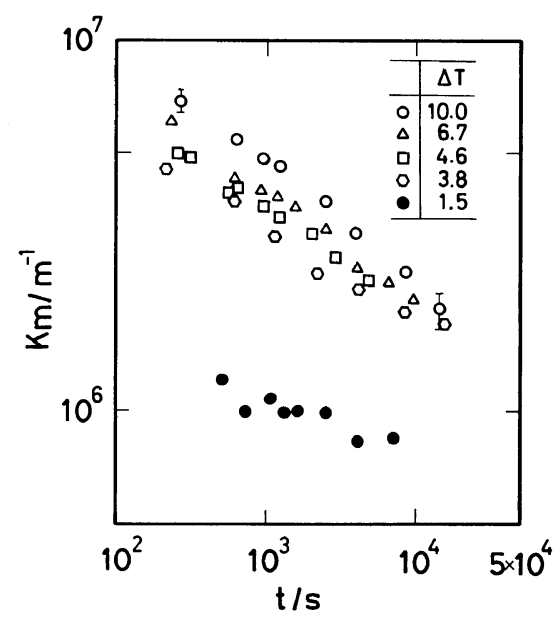

Figure 8. The wave number for $I_{\mathrm{m}}, k_{\mathrm{m}}$, as a function of $t$ for a blend of $50.4 \mathrm{wt} \% \mathrm{PS}$ at various $\Delta T$.

comparable $\Delta T$ are larger for lower PS concentration.

The time dependence of $k_{\mathrm{m}}$ on 50.4 and $23.9 \mathrm{wt} \%$ PS at different $\Delta T$ is shown in Figures 8 and 9 , respectively. In either blend, except for $\Delta T=1.5^{\circ} \mathrm{C}$ at $50.4 \mathrm{wt} \% \mathrm{PS}$, the data points for individual $\Delta T$ approximately follow a straight line. However, the dependence of $k_{\mathrm{m}}$ on $\Delta T$ at fixed times is reversed in the two blends; $k_{\mathrm{m}}$ for $23.9 \mathrm{wt} \%$ PS decreases with increasing $\Delta T$, while that for $50.4 \mathrm{wt} \%$ PS increases with increasing $\Delta T$.

From what we have shown above, the time 
Phase Separation Process in Polymer Systems. I.

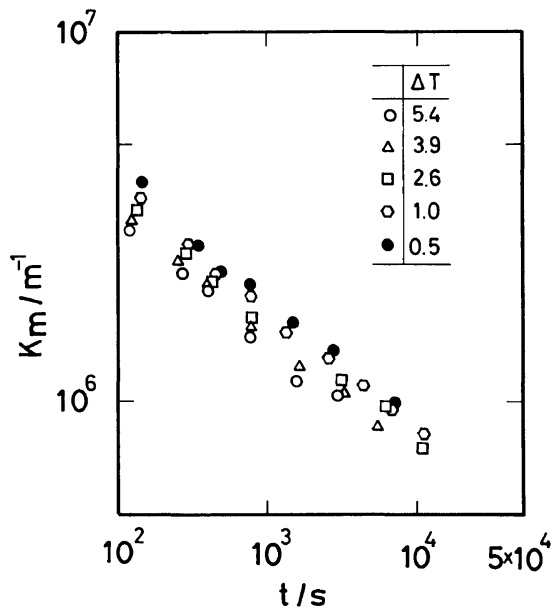

Figure 9. The wave number for $I_{\mathrm{m}}, k_{\mathrm{m}}$, as a function of $t$ for a blend of $23.9 \mathrm{wt} \%$ PS.

changes of $I_{\mathrm{m}}$ and $k_{\mathrm{m}}$ can be described approximately by power-law relations $I_{\mathrm{m}} \propto t^{\theta}$ and $k_{\mathrm{m}} \propto t^{\phi}$. We have found that the same was true for the blends of other concentrations $c$ studied. Table I summarizes the values of $\theta$ and $\phi$ obtained from the present study, and shows that $\theta=0.69( \pm 0.17)$ and $\phi=$ $-0.29( \pm 0.05)$ for all $c$ and $\Delta T$, with a few exceptions indicated by the dashed lines in Table I for which the power-law relations were not observed.

\section{DISCUSSION}

On the basis of the reasons that follow, we concluded that the phase separation investigated in the present study occurred by spinodal decomposition except for a few cases at small $\Delta T$. (i) The cloud point curve is generally located between the spinodal and binodal. ${ }^{38}$ In liquid mixtures of small molecules the cloud point should be closer to the binodal, but in polymer blends it may be rather close to the spinodal because of the low mobility of polymers. In fact, our preliminary measurements of the osmotic compressibility and the correlation length above the cloud point indicated that the spinodal point for our blend was only a few degrees below the cloud point curve even at as low a PS concentration as $10 \mathrm{wt} \%$. Thus we may consider that except at small $\Delta T$ the phase separations in the present study were carried out at temperatures below the spinodal, i.e., in the unstable region. (ii)
Table I. The values of $\phi$ and $\theta$ in power-law expression for $k_{\mathrm{m}}$ and $I_{\mathrm{m}}$ against $t$ : $k_{\mathrm{m}} \propto t^{\phi}$ and $I_{\mathrm{m}} \propto t^{\theta}$.

\begin{tabular}{|c|c|c|c|}
\hline$c / \mathrm{wt} \% \mathrm{PS}$ & $\Delta T$ & $\theta$ & $\phi$ \\
\hline \multirow{5}{*}{23.9} & 5.4 & 0.80 & -0.34 \\
\hline & 3.9 & 0.61 & -0.33 \\
\hline & 2.6 & 0.61 & -0.32 \\
\hline & 1.0 & 0.72 & -0.32 \\
\hline & 0.5 & 0.80 & -0.34 \\
\hline \multirow{5}{*}{39.5} & 7.7 & 0.51 & -0.30 \\
\hline & 5.1 & 0.54 & -0.28 \\
\hline & 3.7 & 0.56 & -0.28 \\
\hline & 2.2 & 0.68 & -0.28 \\
\hline & 0.9 & 0.68 & -0.31 \\
\hline \multirow{5}{*}{50.4} & 10.0 & 0.76 & -0.32 \\
\hline & 6.7 & 0.84 & -0.30 \\
\hline & 4.6 & 0.88 & -0.27 \\
\hline & 3.8 & 0.72 & -0.24 \\
\hline & 1.5 & - & - \\
\hline \multirow{5}{*}{54.9} & 10.0 & 0.74 & -0.26 \\
\hline & 6.7 & 0.84 & -0.28 \\
\hline & 4.8 & 0.74 & -0.24 \\
\hline & 3.6 & - & - \\
\hline & 1.0 & - & - \\
\hline
\end{tabular}

The range of PS concentration studied included the critical concentration at which phase separation is known to proceed by spinodal decomposition whatever the value of $\Delta T$ may be. Our data showed the power-laws $I_{\mathrm{m}} \propto t^{\theta}$ and $k_{\mathrm{m}} \propto t^{\phi}$ to hold over the experimental ranges except at small $\Delta T$ as well as near the critical concentration. (iii) The deviations from these relations at small $\Delta T$ were similar to those observed in mixtures of small molecules, which separate into phases by nucleation and growth mechanism. ${ }^{25}$

\section{Comparison with the Cahn Theory}

$\mathrm{Cahn}^{7}$ derived a diffusion equation for the kinetics of spinodal decomposition, assuming the Ginzburg-Landau type free energy $F(c)$ for a nonequilibrium system $^{8}$ :

$$
F(c)=\int\left[f(c)+K(\nabla c)^{2}\right] \mathrm{d} V
$$

where $f(c)$ is the free energy density of the homogeneous phase and $K(\nabla c)^{2}$ represents the contribution from concentration gradients, with $K$ a 
positive constant. He obtained

$$
\tilde{c}(k, t)=\tilde{c}(k, 0) \exp [R(k) t]
$$

for the Fourier component $\tilde{c}(k, t)$ of local concentration fluctuations at time $t$. In this, $R(k)$ is an amplification factor expressed by

$$
R(k)=k^{2} M\left[-\left(\partial^{2} f / \partial c^{2}\right)_{c_{0}}-2 K k^{2}\right]
$$

where $c_{0}$ is the initial concentration and $M$ is a mobility parameter defined phenomenologically. The function $R(k)$ has a maximum at a $k$ value given by

$$
\begin{aligned}
k_{\mathrm{m}} & =(1 / 2)\left[-\left(\partial^{2} f / \partial c^{2}\right)_{c_{0}} / K\right]^{1 / 2} \\
& =(1 / 2)(-D / M K)^{1 / 2}
\end{aligned}
$$

where $D$ is the diffusion constant defined by

$$
D=M\left(\partial^{2} f / \partial c^{2}\right)_{c_{0}}
$$

Note that the derivative $\left(\partial^{2} f / \partial c^{2}\right)_{c_{0}}$ is negative in the region enclosed by the spinodal. The quantity $k_{\mathrm{m}}$ corresponds to the fastest mode of growth of concentration fluctuations in the spinodal decomposition. With the Born approximation, the scattered intensity $I(k, t)$ for wave number $k$ and time $t$ can be obtained from eq 3 to be

$$
I(k, t)=I(k, 0) \exp [2 R(k) t]
$$

which also has a maximum at $k_{\mathrm{m}}$ given by eq 5 .

The characteristic results from the Cahn theory are that $k_{\mathrm{m}}$ is independent of time $t$, while $I_{\mathrm{m}}$ increases exponentially with $t$. These are, however, at variance with the present experimental results shown in Figures 6 through 9, which indicate that $k_{\mathrm{m}}$ decreases with $t$ as $k_{\mathrm{m}} \propto t^{\phi}$ and $I_{\mathrm{m}} \propto t^{\theta}$. This discrepancy may be attributed to the fact that the processes observed in our study do not correspond to the early stage behavior described by the linear theory of Cahn.

Though not in conformity with the Cahn theory, the present data at short times may permit an approximate evaluation of the parameter in the theory. For this purpose we follow the procedure of Goldburg et al., ${ }^{20}$ and recast eq 4 in the form,

$$
R(k) / k^{2}=-D-2 M K k^{2}
$$

where eq 6 has been substituted. The data for 50.4 $\mathrm{wt} \% \mathrm{PS}$ and $\Delta T=6.7^{\circ} \mathrm{C}$ were subjected to analysis.

First, the data points for different $k$ in Figure 5 are fitted by a smooth curve, and $R(k)$ is determined as the slope of the tangent to each curve at fixed times (see eq 7). The values of $R(k) / k^{2}$ so obtained at $t=610$ and $1560 \mathrm{~s}$ are plotted against $k^{2}$ in Figure 10. The resulting plots are not linear, which is at variance with the prediction of the Cahn theory (eq 8 ). We approximate the solid curves in Figure 10 by the dot-dash lines connecting two symmetrical points with respect to $k_{\mathrm{m}}$ (indicated by the arrows in the figure), and compare these lines with eq 8 to evaluate $D$ and $M K$. The values of $D, M K$, and $k_{\mathrm{m}}$ so obtained for $t=610$ and $1560 \mathrm{~s}$ are given in Table II, where $k_{\mathrm{m}}$ was calculated by eq 5 and $k_{\mathrm{m}}(\exp )$ is the directly determined $k_{\mathrm{m}}$ value (see Figure 4). The values of $D$ and $M K$ are roughly $-1 \times 10^{-16} \mathrm{~m}^{2} \mathrm{~s}^{-1}$ and $1 \times 10^{-30} \mathrm{~m}^{4} \mathrm{~s}^{-1}$, respectively.

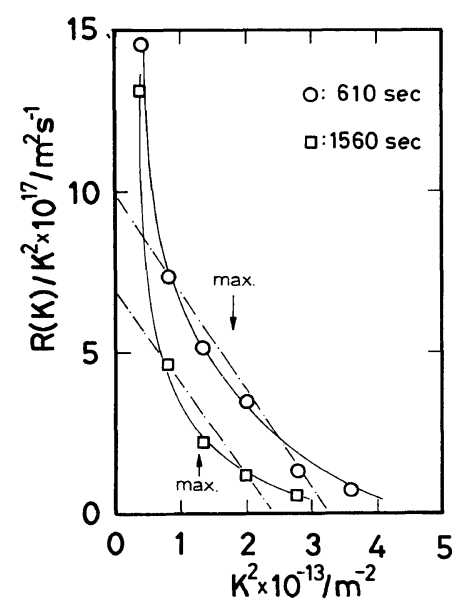

Figure 10. The values of $R(k) / k^{2}$ as a function of $k^{2}$ at selected values of $t$.

Table II. The parameters $D, M K$, and $k_{\mathrm{m}}$ obtained from the Cahn theory for the blend of $50.4 \mathrm{wt} \%$ PS

\begin{tabular}{rrrrr}
\hline$t / \mathrm{s}$ & $D / \mathrm{m}^{2} \mathrm{~s}^{-1}$ & $M K / \mathrm{m}^{4} \mathrm{~s}^{-1}$ & $k_{\mathrm{m}} / \mathrm{m}^{-1}$ & $k_{\mathrm{m}}(\exp ) / \mathrm{m}^{-1}$ \\
\hline 610 & $-1 \times 10^{-16}$ & $1.4 \times 10^{-30}$ & $4.2 \times 10^{6}$ & $4.25 \times 10^{6}$ \\
1560 & $-7 \times 10^{-17}$ & $1.4 \times 10^{-30}$ & $3.5 \times 10^{6}$ & $3.57 \times 10^{6}$ \\
\hline
\end{tabular}


By pulsed NMR and microscopic observations, Nishi et al. ${ }^{30}$ obtained $D=-2.8 \times 10^{-17} \mathrm{~m}^{2} \mathrm{~s}^{-1}$ and $M K=2.57 \times 10^{-31} \mathrm{~m}^{4} \mathrm{~s}^{-1}$ for a mixture of PS and poly(vinyl methyl ether), which are consistent with ours in order of magnitude.

The value of $D$ should vary widely with $\Delta T$ or the distance from the spinodal on which it vanishes. According to eq 5 of the Cahn theory, $k_{\mathrm{m}}$ is independent of time and decreases at any concentration as the temperature approaches the spinodal point. This prediction was confirmed for the blend of poly(2,6-dimethyl-1,4-phenylene oxide) and caprolactam by van Aartsen et al., ${ }^{27}$ who obtained $k_{\mathrm{m}}$ ranging $6 \times 10^{6}-9 \times 10^{6} \mathrm{~m}^{-1}$, which is consistent with our values of $k_{\mathrm{m}}$. However, our study demonstrated that $k_{\mathrm{m}}$ decreases with time and either increases or decreases with $\Delta T$ at fixed times, depending on the concentration of the blend, as can be seen in Figures 8 and 9. This time effect must be taken into account when we consider the dependence of $k_{\mathrm{m}}$ on $\Delta T$. At a fixed time after quenching of a blend, we should be able to observe a more advanced stage of phase separation for larger $\Delta T$, since the phase separation develops faster as $\Delta T$ is increased. Therefore, when the time dependence of $k_{\mathrm{m}}$ as observed here is present, it should make $k_{\mathrm{m}}$ for larger $\Delta T$ smaller when compared at a fixed time. On the other hand, according to the Cahn theory, $k_{\mathrm{m}}$ at a fixed degree of phase separation is larger for larger $\Delta T$. These two competitive effects complicate the dependence of $k_{\mathrm{m}}$ on $\Delta T$.

\section{Comparison with Other Theories}

Langer, Bar-on, and Miller ${ }^{12}$ developed a statistical theory of phase separation (LBM theory) by taking thermal fluctuations into account and by incorporating non-linear terms in eq 2 to describe the stage after the linear region treated by Cahn. The theory was subjected to a numerical study using a new computational technique. ${ }^{12}$ The results obtained are fitted by a power-law expression $q_{\mathrm{m}} \propto \tau^{\phi}$ with $\phi=-0.21$, where $q_{\mathrm{m}}=\xi k_{\mathrm{m}}$ and $\tau=t /\left(\xi^{2} / D\right)$, which are reduced $k_{\mathrm{m}}$ and $t$, respectively, with $\xi$ the correlation length. Thus, the LBM theory is consistent with our observed power-law relation between $k_{\mathrm{m}}$ and $t$, and the predicted $\phi$ is not very different from the values in Table I.

By developing a new theory of phase separation on the basis of cluster dynamics, Binder et al. ${ }^{16}$ showed that the power-law relations $k_{\mathrm{m}} \propto t^{\phi}$ and
$I_{\mathrm{m}} \propto t^{\theta}$ with $\phi=-1 / 3$ and $\theta=1$ hold at early stages of spinodal decomposition in liquid mixtures. This value of $\phi$ is much closer to our experimental results than that predicted by the LBM theory. However, as for the value of $\theta$ the agreement between theory and experiment is not as good as this.

\section{Comparison with the Results for Binary Mixtures of Small Molecules}

Wong and Knobler $^{24,25}$ recently investigated phase separation of mixtures of isobutyric acid and water in the critical region, and found that the time dependence of $k_{\mathrm{m}}$ and $I_{\mathrm{m}}$ were represented by the power-law relations both for the critical and offcritical mixtures quenched into the unstable state. The exponents $\phi$ and $\theta$ were dependent on the quench depth for the critical mixture, but were substantially constant for the off-critical mixtures: $\phi=-0.29--0.35$ and $\theta=1.08-1.21$. Though this value of $\theta$ is slightly larger than our value, the power-law variations of $k_{\mathrm{m}}$ and $I_{\mathrm{m}}$ with time are consistent with our results. Wong and Knobler also showed that $I_{\mathrm{m}}$ of the mixture quenched to the metastable state increased roughly in proportion to $t^{2}$ at first and to $t^{1 / 2}$ at subsequent stages, instead of obeying the power-law. This behavior is analogous to that we observed for small $\Delta T$ in our system (see Figure 6). These coincidences, though not entirely quantitative, suggest that there is no essential difference in the mechanism of phase separation between mixtures of small molecules and polymer blends.

Goldburg et al. ${ }^{18-23}$ made extensive studies on the critical mixture of 2-6 lutidine and water and showed that $I_{\mathrm{m}}$ and $q_{\mathrm{m}}$ for different $\Delta T$ plotted against reduced separation time $\tau$ yielded a single composite curve, in qualitative agreement with the LBM theory and the cluster-dynamic theory. However, the rate of increase in $I_{\mathrm{m}}$ and that of decrease in $k_{\mathrm{m}}$ were more rapid than expected theoretically at large $t$. This discrepancy was explained by Goldburg et al., who considered long range hydrodynamic interactions in the critical region according to Kawasaki and Ohta. ${ }^{17}$ Our results are consistent with their data except the rapid growth of $I_{\mathrm{m}}$ and $k_{\mathrm{m}}^{-1}$ at large $t$. Chou and Goldburg $^{23}$ reported an empirical relation between $\phi$ and $\theta$ or between $I_{\mathrm{m}}$ and $k_{\mathrm{m}}$, which reads $\theta=-3 \phi$ or $I_{\mathrm{m}} k_{\mathrm{m}}{ }^{3}=$ constant, for spinodal decomposition. They deduced this relation by a geometrical consideration. Table I indicates that our data on 50.4 


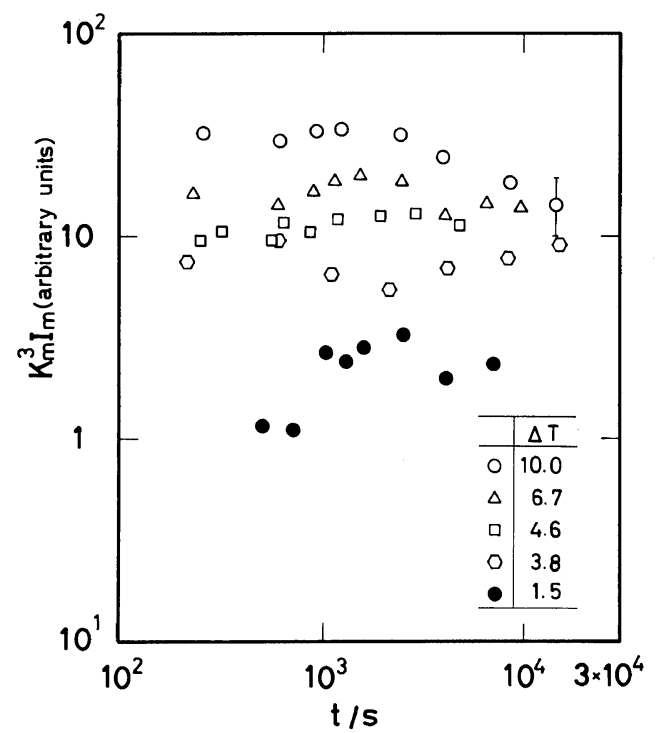

Figure 11. The product of $I_{\mathrm{m}}$ and $k_{\mathrm{m}}{ }^{3}$ as a function of $t$.

and $54.9 \mathrm{wt} \%$ PS blends approximately satisfy $\theta=$ $-3 \phi$. In Figure 11, the values of $I_{\mathrm{m}} k_{\mathrm{m}}{ }^{3}$ for $50.4 \mathrm{wt} \%$ PS are plotted against time for various values of $\Delta T$. It is seen that $I_{\mathrm{m}} k_{\mathrm{m}}{ }^{3}$ is approximately constant except at small $\Delta T$.

In conclusion, there is no essential difference in the mechanism of phase separation between mixtures of small molecules and polymer blends.

\section{REFERENCES}

1. E. D. Siggia, Phys. Rev., A20, 595 (1979).

2. J. Zarzycki, Disc. Faraday Soc., 50, 122 (1971).

3. I. M. Lifshitz and V. V. Slyozov, J. Phys. Chem. Solids, 19, 35 (1961).

4. C. Wagner, Zeit. Elektochemie, 65, 581 (1961).

5. J. W. Cahn, Trans. Metall. Soc. AIME, 242, 166 (1968).

6. M. Hillert, Acta Metall., 9, 525 (1961).

7. J. W. Cahn, J. Chem. Phys., 42, 93 (1965).

8. J. W. Cahn and J. E. Hilliard, J. Chem. Phys., 28, 258 (1958).

9. H. E. Cook, Acta Metall., 18, 297 (1970).

10. J. S. Langer, Ann. Phys., 65, 53 (1971).
11. J. S. Langer, Acta Metall., 21, 1649 (1973).

12. J. S. Langer, M. Bar-on, and H. D. Miller, Phys. Rev., A11, 1417 (1975).

13. F. F. Abraham, J. Chem. Phys., 63, 157 (1975).

14. F. F. Abraham, J. Chem. Phys., 63, 1316 (1975).

15. P. G. de Gennes, J. Chem. Phys., 72, 4756 (1980).

16. K. Binder and D. Stauffer, Phys. Rev. Lett., 33, 1006 (1974).

17. K. Kawasaki and T. Ohta, Prog. Theor. Phys., 59, 362 (1978).

18. W. I. Goldburg and J. S. Huang, "Fluctuations, Instabilities and Phase Transitions," T. Riste, Ed., Plenum Press, New York, N. Y., 1975, p 87.

19. J. S. Huang, W. I. Goldburg, and A. W. Bjerkaas, Phys. Rev. Lett., 32, 921 (1974).

20. A. J. Schwartz, J. S. Huang, and W. I. Goldburg, J. Chem. Phys., 62, 1874 (1975).

21. W. I. Goldburg, C. H. Shaw, J. S. Huang, and M. S. Pilant, J. Chem. Phys., 68, 484 (1978).

22. W. I. Goldburg, A. J. Schwartz, and M. W. Kim, Prog. Theor. Phys. Suppl., 64, 477 (1978).

23. Y. C. Chou and W. I. Goldburg, Phys. Rev., A20, 2105 (1979).

24. N. C. Wong and C. M. Knobler, J. Chem. Phys., 66, 4707 (1977).

25. N. C. Wong and C. M. Knobler, J. Chem. Phys., 69, 725 (1978).

26. J. Wenzel, U. Limbach, G. Bresonic, and G. M. Schneider, J. Phys. Chem., 84, 1991 (1980).

27. J. J. van Aartsen and C. A. Smolders, Eur. Polym. J., 6, 1105 (1970).

28. C. A. Smolders, J. J. van Aartsen, and A. Steenbergen, Kolloid Z. Z. Polym., 243, 14 (1971).

29. P. T. van Emmerik, C. A. Smolders, and W. Geymayer, Eur. Polym. J., 9, 309 (1973).

30. T. Nishi, T. T. Wang, and T. K. Kwei, Macromolecules, 8, 227 (1975).

31. V. M. Andreyeva, A. A. Tager, I. S. Tyukova, and L. F. Golenkova, Polym. Sci. U.S.S.R., 19, 3005 (1978).

32. L. P. McMaster, Adv. Chem. Ser.; 142, 43 (1975).

33. G. G. A. Böhm, K. R. Lucas, and W. G. Mayes, Rubber Chem. Technol., 50, 714 (1977).

34. S. Nojima and T. Nose, to be published.

35. H. Hocker, G. J. Blake, and P. J. Flory, Trans. Faraday Soc., 67, 2251 (1971).

36. "Polymer Handbook," J. Brandrup and E. H. Immergut Ed., Wiley, New York, N. Y., 1975, V 60.

37. M. Bank, J. Leffingwell, and C. Thies, Macromolecules, 4, 43 (1971).

38. S. Krishnamurthy and W. I. Goldburg, Phys. Rev., A22, 2147 (1980). 